\title{
X-ray Observations of Poor Groups
}

\author{
John S. Mulchaey \\ The Observatories of the Carnegie Institution of Washington, 813 Santa \\ Barbara St., Pasadena, CA 91101
}

\begin{abstract}
X-ray observations of groups have provided considerable new insight into these cosmologically important systems. Recent ROSAT surveys of loose and compact groups suggest that $\sim 50 \%$ of all nearby groups contain a hot intragroup medium, including many spiral-rich groups. However, spiral-only groups tend not to be X-ray detected. Spectroscopic studies of X-ray groups indicate that these systems contain a large number of faint galaxies. The existence of a large population of faint galaxies, combined with the extended X-ray halos, is consistent with Xray detected groups being real, bound systems. The nature of non-X-ray detected groups, which do not contain an extensive faint galaxy population, is still unclear.

ROSAT observations have also revealed the existence of individual elliptical galaxies with X-ray properties and total masses similar to those of X-ray groups. These objects may be the merger remnants of X-ray groups or 'failed groups' (i.e. overdensities that formed only a single galaxy). The number density of these objects suggests they make a nonnegligible contribution to the mass density of the universe.
\end{abstract}

\section{Introduction}

Although most galaxies in the local universe belong to small groups, we know surprisingly little about these systems. This is because group studies are hampered by small number statistics: a typical poor group contains only a few bright galaxies. Some of the outstanding questions regarding these systems include: 1) Are groups bound physical systems or simply chance superpositions of galaxies along the line of sight? 2) What is the typical mass of a group? 3) How do groups contribute to the mass density of the universe? 4) How is the evolution of galaxies different in groups than in rich clusters? and 5) How have groups evolved in time?

The discovery that many groups are X-ray sources has provided new insight into these important systems. ROSAT observations indicate that the X-ray emission in groups is spatially extended, often beyond the apparent optical extent of the group (see Figure 1). X-ray spectroscopy suggests the emission mechanism is most likely thermal bremsstrahlung. This interpretation requires that the entire volume of the group be filled with a hot, low density gas. This extended gas component is referred to as the intragroup medium in analogy to the X-ray emitting intracluster medium found in rich clusters of galaxies. The X-ray data 


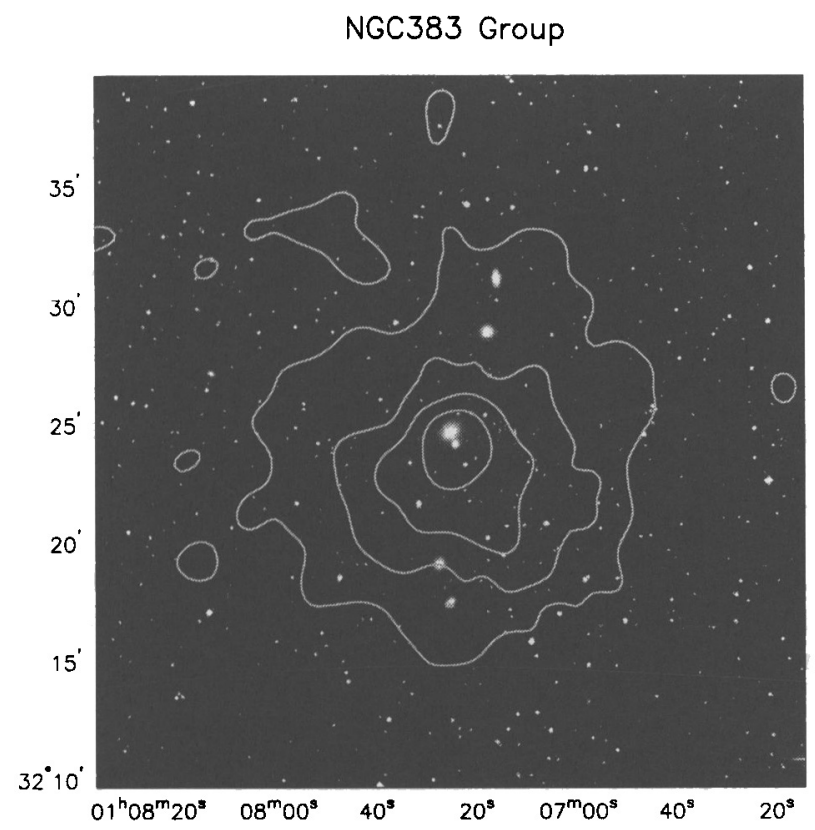

Figure 1. Contours of the X-ray emission (ROSAT PSPC) in the NGC 383 group overlayed on an optical image of the group. The coordinate scale is for epoch J2000. One arcminute corresponds to 14.4 $\mathrm{h}_{100}{ }^{-1} \mathrm{kpc}$ at the assumed distance to the group.

can be used to derive the following properties of the intragroup medium: 1) the global gas temperature; 2) the projected temperature as a function of radius; 3 ) the projected gas density as a function of radius and 4) the "metallicity" of the gas, derived from the strengths of emission lines of abundant elements like iron, oxygen, silicon and sulfur. With these observables and assumptions about the state of the hot gas, we can probe the dynamics of groups and the star formation history of the group galaxies.

\section{ROSAT Surveys of Groups}

Unfortunately, the realization that X-ray observations are such a valuable probe of poor groups came relatively late in the lifetime of the ROSAT PSPC. Therefore, large systematic surveys of groups were not carried out with this instrument. However, the ROSAT PSPC did observe many galaxies and because most galaxies occur in groups, many groups were observed serendipitously.

In the end, over 100 nearby groups were observed by the ROSAT PSPC during its lifetime including a significant fraction of the Hickson Compact Groups (HCGs; Hickson 1982) and a large number of more typical loose groups. Ponman et al. (1996) combined pointed PSPC observations with much more shallow 
ROSAT All-Sky Survey data to produce an almost complete survey of the HCGs. These authors detect extended X-ray emission in $\sim 26 \%$ (22 of 85 groups) of the systems studied and infer that $\sim 75 \%$ of the HCGs contain a hot intragroup medium (when one corrects for the detection limits of most of the observations). However, given the compactness of these groups, the nature of the X-ray emission in some of the detected HCGs is far from clear. For example, although Stephan's Quintet (HCGs) is extended in the ROSAT PSPC data, a higher resolution ROSAT HRI image suggests that most of the extended emission is associated with a shock feature and not with hot intragroup gas (Pietsch et al. 1997; Sulentic, this volume). Another very interesting case, discussed extensively during this meeting is HCG 16 . The X-ray emission is extremely clumpy in this group and appears concentrated around the brightest group members, most of which display nuclear activity or are undergoing active star formation (Ribeiro et al. 1996). Given the unusually high level of activity in this group, it is tempting to associate much of the observed X-ray emission with the galaxies. However, as noted by Trevor Ponman during this meeting, the X-ray to infrared luminosity ratio of this system is much higher than one would expect if the X-ray emission is related to the galaxies' activity. Higher spatial resolution observations with CHANDRA should help elucidate the nature of the X-ray emission in HCGs.

Many of the problems inherent to the study of compact groups can be avoided with loose group samples. Mulchaey et al (1996) studied a sample of 14 loose groups selected from optical catalogs and found diffuse X-ray emission in four systems $(\sim 29 \%)$. Helsdon \& Ponman (1999) studied an archival sample of 35 loose groups selected from X-ray and optical studies (their sample also contains two X-ray bright HCGs). Considering only those groups from the optically-selected sample, $50 \%$ (12 of 24) of the groups contain a hot intragroup medium. In collaboration with David Davis (MIT), Richard Mushotzky (NASA/GSFC) and David Burstein (Arizona State), I have studied a sample of 57 optically-selected loose groups observed with the ROSAT PSPC. We find a diffuse X-ray component in approximately $50 \%$ (27 of 57 ) of the systems studied (note that all 24 of the optically-selected groups in Helsdon \& Ponman (1999) are also in our sample). Based on these surveys, it appears that approximately half of the groups in local redshift surveys contain a hot X-ray emitting intragroup medium.

A correlation between the presence of X-ray emission and the morphological composition of groups was suggested by the earliest ROSAT group surveys. Ebeling et al. (1994) were the first to claim such an effect, noting that all but one of the X-ray detected Hickson Compact groups in the ROSAT All-Sky Survey data had spiral fraction less than $50 \%$. Subsequent studies of small samples appeared to support this trend (Henry et al. 1995; Pildis et al. 1995; Mulchaey et al. 1996). However, Ponman et al. (1996) came to a very different conclusion based on their much larger survey of the HCGs. They detected several groups with high spiral fractions and offer as an extreme example HCG 16, a compact group that contains only spirals.

Figure 2 shows the distribution of early-type fraction for X-ray detected groups (shaded histogram) and all group studied by ROSAT (open histogram). As is immediate apparent from these histograms, a significant number of spiral- 


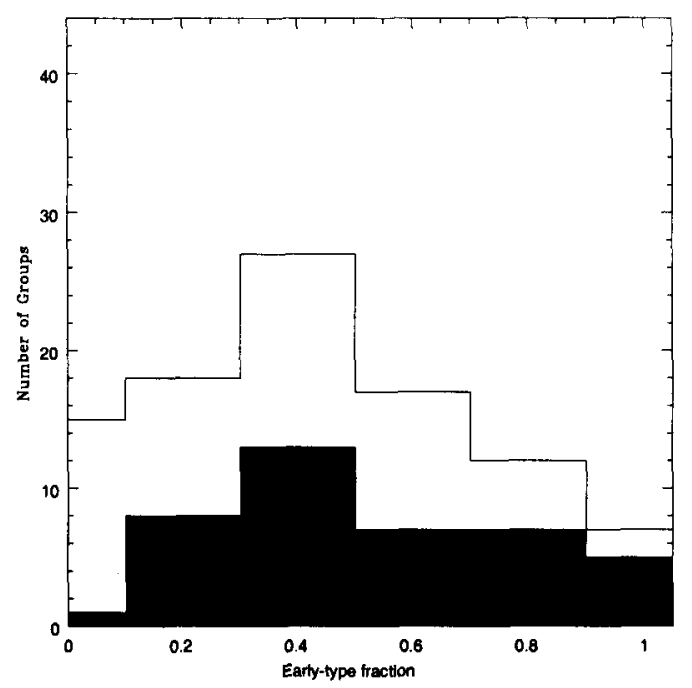

Figure 2. Histogram of the early-type fraction for X-ray detected groups (shaded) and for all groups studied by ROSAT to date (open).

rich groups (i.e. early-type fraction less than 0.5 ) do contain diffuse $\mathrm{X}$-ray emission, confirming the conclusion of Ponman et al. (1996).

A closer examination of Figure 2 reveals that while many spiral-rich systems are X-ray sources, spiral-only groups tend not to contain a diffuse X-ray component. The one exception is the peculiar group discussed earlier, HCG 16 . All of the other X-ray detected groups in Figure 2 contain at least one earlytype galaxy. In fact, in nearly all of the X-ray detected groups the brightest group galaxy is an elliptical galaxy (Ebeling et al. 1994; Mulchaey et al. 1996; Mulchaey \& Zabludoff 1998).

\section{Optical Properties of X-ray Groups}

To probe the dynamical state of groups in more detail, Ann Zabludoff (Univ. of Arizona) and I have undertaken a study of the faint galaxy population in a sample of X-ray and non-X-ray detected groups (Zabludoff \& Mulchaey 1998; Mulchaey \& Zabludoff 1998). For each group in our sample, we have used the multi-fiber spectrograph (Shectman et al. 1992) on the du Pont $2.5 \mathrm{~m}$ telescope at the Las Campanas Observatory to obtain redshifts for the $\sim 100$ brightest galaxies in the $1.5 \times 1.5$ degree field centered on each group.

Figure 3 shows the resulting galaxy velocity distributions for four X-ray detected groups (top two panels) and two non-X-ray detected groups (bottom panel). The galaxies we identify as group members are indicated by the dashed histograms. These histograms indicate that the X-ray groups contain at least 
20-50 group members down to absolute magnitudes $M_{B} \sim(-14$ to -16$)+5 \log _{10}$ $\mathbf{h}_{100}$. The large number of group members, many of which are early-type galaxies, combined with the presence of X-ray emission, argues that X-ray groups are real, physical systems. Given the large number of group velocities we measure, we are also able to determine reliable velocity dispersions for these groups for the first time. The velocity dispersions of the X-ray groups range from $\sim 200-500$ $\mathrm{km} \mathrm{s}^{-1}$. We also find that the velocity dispersions of these groups calculated using the five brightest galaxy velocities only (as if often the case for groups in the literature) tends to underestimate the true velocity dispersion because the tails of the galaxy velocity distribution are not well-sampled (Zabludoff \& Mulchaey 1998). In the worst case in our sample (the NGC 2563 group), the five-member velocity dispersion underestimates the true value by a factor of three.

In contrast to X-ray groups, non-X-ray detected groups tend to have fewer than 10 galaxy members down to the same absolute magnitude limits. Because we do not detect diffuse X-ray emission or a significant fainter population in the non-X-ray detected groups, the nature of these systems is still unclear. We do find, however, that the non-X-ray groups, which consist of one or two $L^{*}$ or brighter spirals with several fainter galaxies that may be satellites, are morphologically akin to the Local Group (although our samples are not sufficiently deep to ascertain whether any group has a dwarf spheroidal population like that of the Local Group; van den Bergh 1992). If the non-X-ray groups are dynamically similar to the Local Group, they are bound (see Zaritsky 1994). Alternatively, these groups may be chance superpositions or unbound galaxies in filaments along the line-of-sight (Hernquist et al. 1995).

\section{Single Galaxy Groups}

Traditionally, a group is defined as a galaxy system containing several luminous galaxies. Recent $\mathrm{X}$-ray observations suggest that this might not be the best way to define such systems. In particular, several individual galaxies with very luminous X-ray halos have been discovered (see Fig. 4; Ponman et al 1994.; Mulchaey \& Zabludoff 1999; Vikhlinin et al. 1999). The temperature, extent and luminosity of these objects are comparable to those of X-ray groups. The total masses inferred from the X-ray data are also similar to those of X-ray groups. Therefore, from an X-ray and mass perspective, these objects should be thought of as groups. However, they clearly fail the classic definition of a group.

The existence of single-galaxy X-ray groups was predicted by Ponman \& Bertram (1993) after they discovered a large X-ray halo around HCG 62. These authors noted that the merging timescale for the big galaxies in a compact group like HCG 62 is typically less than a Hubble time. However, the cooling time for the intragroup medium is significantly longer than a Hubble time (outside of the central group core). Therefore, the galaxies in some groups may have had enough time to merge into a single giant elliptical galaxy, while the properties of the X-ray halo will remain basically unchanged. Another possibility is that the single-galaxy groups are 'failed groups', a local overdensity in which other bright galaxies never formed (Mulchaey \& Zabludoff 1999).

Regardless of the origin of these objects, they are a reminder that galaxies are not always a good tracer of mass. Vikhlinin et al. (1999) have estimated the 


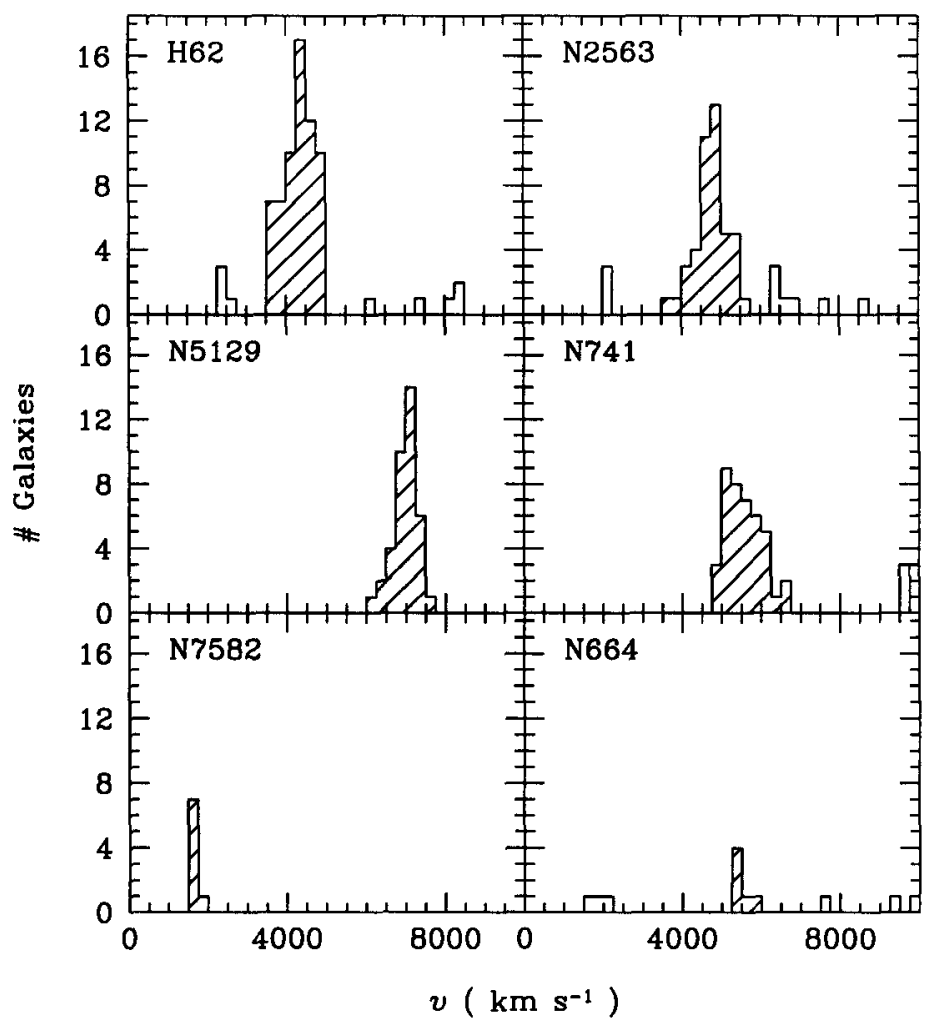

Figure 3. Galaxy velocity distributions for four X-ray detected groups (top two panels) and two non-X-ray detected groups (bottom panel). The dashed histograms indicate the galaxies that we identify as group members. In this Figure, we only show velocities in the vicinity of the groups. For the complete velocity histograms see Zabludoff \& Mulchaey (1998). 
NGC 1132

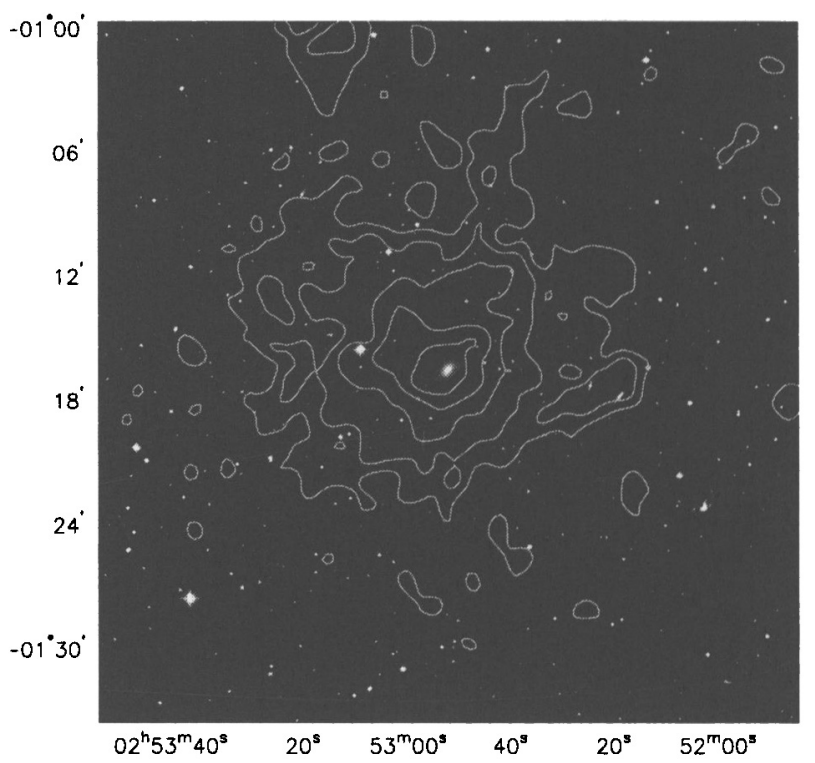

Figure 4. Contour map of the X-ray emission (ASCA) in the isolated elliptical NGC 1132 (Mulchaey \& Zabludoff 1999). The X-ray properties of this system are consistent with those found for luminous $\mathrm{X}$-ray groups. The coordinate scale is for epoch J2000. One arcminute corresponds to $19.4 \mathrm{~h}_{100}{ }^{-1} \mathrm{kpc}$ at the assumed distance to the galaxy. 
number density of these objects from their survey of deep ROSAT pointings and find that single-galaxy groups comprise $\sim 20 \%$ of all X-ray luminous groups. Thus, these objects make a non-negligible contribution to the total mass density of the universe. Future surveys with CHANDRA and XMM should provide new insight into the nature of this class of objects.

\section{References}

Helsdon, S. F., \& Ponman, T. J. 1999, MNRAS, in press.

Henry, J. P., Gioia, I. M., Huchra, J. P., Burg, R., \& McLean, B. 1995, ApJ, 449,422

Hernquist, L., Katz, N. \& Weinberg, D. 1995, ApJ, 442, 57

Hickson, P. 1982, ApJ, 255, 382

Mulchaey, J. S., Davis, D. S., Mushotzky, R. F., \& Burstein, D. 1996, ApJ, 456, 80

Mulchaey, J. S., Zabludoff, A. I., 1998, ApJ, 496, 73

Mulchaey, J. S., Zabludoff, A. I., 1999, ApJ, 514, 133

Pietsch, W., Trinchieri, G., Arp, H., Sulentic, J. W., 1997, AA, 322, 89

Pildis, R. A., Bregman, J. N., Evrard, A. E., 1995, ApJ, 443, 514

Ponman, T. J., Allan, D. J., Jones, L. R., Merrifield, M., McHardy, I. M., 1994, Nature, 369, 462

Ponman, T. J., Bertram, D., 1993, Nature, 363, 51

Ponman, T. J., Bourner, P. D. J., Ebeling, H., Böhringer, H., 1996, MNRAS, 283,690

Ribeiro, A. L. B., de Carvalho, R. R., Coziol, R., Capelato, H. V., \& Zepf, S. E., 1996, ApJ, 463, 5

Shectman, S. A., Landy, S. D., Oemler, A., Tucker, D. L., Lin, H., Kirshner, R. P., Schechter, P. L. 1996, ApJ, 470, 172

van den Bergh, S. 1992, AA, 264, 75

Vikhlinin, A, McNamara, B. R., Hornstruo, A., Quintana, H., Forman, W., Jones, C., \& Way, M. 1999, ApJ, 520, L1

Zabludoff, A. I., Mulchaey, J.S., 1998, ApJ, 496, 39

Zaritsky, D. 1994, in The Local Group: Comparative and Global Properties, eds. A. Layden, R. C. Smith, and J. Storm, ESO Conference and Workshop Proceedings No. 51, p.187 